\title{
Quantitative Dynamic Contrast-Enhanced Magnetic Resonance Imaging in Symptomatic Uterine Fibroids and Normal Uterus: A Feasibility Study
}

\author{
Soheila Majd, ${ }^{1}$ Basri Johan Jeet Abdullah,, ${ }^{1,}$ Sazilah Ahmad Sarji, ${ }^{1}$ Chai Hong Yeong, ${ }^{1}$ and Noor Azmi \\ Mat Adenan ${ }^{2}$ \\ ${ }^{1}$ Department of Biomedical Imaging, Faculty of Medicine, University of Malaya, 50603 Kuala Lumpur, Malaysia \\ ${ }^{2}$ Department of Obstetrics and Gynaecology, Faculty of Medicine, University of Malaya, 50603 Kuala Lumpur, Malaysia \\ "Corresponding author: Basri Johan Jeet Abdullah, Department of Biomedical Imaging, Faculty of Medicine, University of Malaya, 50603 Kuala Lumpur, Malaysia. E-mail: \\ basrij@ummc.edu.my
}

Received 2016 January 10; Revised 2016 July 29; Accepted 2016 November 15.

\begin{abstract}
Background: Dynamic contrast enhanced-magnetic resonance imaging (DCE-MRI) is a relatively new imaging technique that provides additional information on vascularity and permeability of the uterine tissues to improve decision making in patient management.

Objectives: The purpose of this study was to obtain the quantitative DCE-MRI parameters of symptomatic uterine fibroids and compare to the parameters obtained from normal myometrium, endometrium, and cervix.

Patients and Methods: Nineteen patients (aged $40 \pm 5$ years) with the clinical diagnosis of uterine fibroids were recruited. After routine MRI pelvis scan, DCE-MRI was performed using T1 volumetric liver acquisition with volume acceleration (LAVA) sequence. The data were post-processed using an independent DCE-MRI analysis software. ROI was drawn on the fibroid and the quantitative DCE-MRI parameters i.e. $K_{\text {trans }}$ (volume transfer between the blood plasma and extracellular fluid), $K_{\text {ep }}$ [flux rate constant between the extracellular extravascular space (EES) and blood plasma], $V_{e}$ (EES fractional volume), and semi-quantitative parameters i.e. TTP (time to peak), and IAUGC (initial area under the gadolinium concentration-time curve) were determined. The patients then underwent hysterectomy and uterine fibroids were confirmed in all the patients by histopathology results. In the control group, fifteen healthy volunteers (aged $32 \pm 6$ years) who did not have uterine fibroid confirmed by sonography examination were recruited. The volunteers underwent the same DCE-MRI scan as the patients and the quantitative DCE-MRI parameters of the normal myometrium, endometrium and cervix were obtained. The DCE-MRI parameters from these two groups were then compared.

Results: The median $K_{\text {trans }}$ in the myometrium, endometrium and cervix of the healthy volunteers were $0.26 \pm 0.13,0.20 \pm 0.15$, and $0.30 \pm 0.13 \mathrm{~min}^{-1}$, respectively. No statistical significant difference was found in all the DCE-MRI parameters between the myometrium, endometrium and cervix of the healthy volunteers. The median $\mathrm{K}_{\text {trans }}$ in fibroids was $0.50 \pm 0.25 \mathrm{~min}^{-1}$. There were statistically significant differences $(\mathrm{P}<0.05)$ found in $\mathrm{K}_{\text {trans }}$ and $\mathrm{V}_{\mathrm{e}}$ between the normal myometrium and fibroid tissue.

Conclusions: The $\mathrm{K}_{\text {trans }}$ and $\mathrm{V}_{\mathrm{e}}$ of fibroid tissue were statistically significantly higher than those obtained from the normal myometrium, whereby the suggested cut-off values were $0.47 \mathrm{~min}^{-1}$ (sensitivity $64.7 \%$, specificity $92.3 \%$ ) and 0.62 (sensitivity $70.6 \%$, specificity 78.6\%), respectively. Quantitative DCE-MRI findings from this study may provide a foundation for assessment of other uterine pathologies.
\end{abstract}

Keywords: Dynamic Contrast-Enhanced Magnetic Resonance Imaging (DEC-MRI), Quantitative, Semi-Quantitative, $\mathrm{K}_{\text {trans }}, \mathrm{V}_{\mathrm{e}}$, Uterine Fibroids, Uterus

\section{Background}

Quantitative dynamic contrast-enhanced magnetic resonance imaging (DCE-MRI) is a gadolinium diethylenetriamine penta-acetic acid (Gd-DTPA) contrast enhanced imaging technique to assess the permeability and vascularity of tissues (1). The enhancement pattern in DCE-MRI also reflects intratumoral angiogenic activity. In oncology, it is an instrumental tool for treatment planning as it provides physiologic information on the tumor in addi- tion to anatomic detail (2). High sensitivity (> 90\%) and moderate specificity ( $>65 \%$ ) have been reported in breast cancer imaging using this technique (3-7). These dynamic MR sequences can be easily incorporated with minimal resource implications i.e. adding less than 10 minutes to the total scanning time. Further, since it does not utilize ionizing radiation, it is an ideal tool for repeat imaging studies in assessing responses to therapy.

Uterine fibroids are the most common benign tumors 
of the female reproductive system. They originate from the smooth muscle wall of the uterus and usually occur in clusters. There are three different types of fibroids based on their anatomical location, i.e. intramural, subserosal, and submucosal fibroids (8). Due to the possibility of severe symptoms such as menorrhagia/hyper-menorrhea and the impact on fertility, it is very important to accurately diagnose and identify the best treatment for uterine fibroids (9). MRI with its superior soft tissue differentiation capabilities gives exquisite information of uterine anatomy; therefore, malignant change may be detected. DCE-MRI provides additional information on vascularity and permeability of the uterine tissues that can aid in decision making for the best treatment option (10). This technique may also potentially be used to evaluate newer drug therapies for uterine fibroids, adenomyosis or uterine sarcoma.

The DCE-MRI protocol generally involves three-step acquisition. First, initial images are acquired to localize the tumor and provide anatomical information. Second, imaging sequences that allow calculation of baseline tissue T1values before contrast agent administration are acquired to enable subsequent analysis. Third, dynamic data are acquired every few seconds in T1-weighted (T1W) images over a period of 5 to 10 minutes (11). DCE-MRI images can be analyzed in quantitative (model-based) and semi-quantitative (non-model based) approaches. The quantitative parameters include the volume transfer coefficient of contrast between blood plasma and extracellular extravascular space (EES) $\left(K_{\text {trans }}\right)$, rate constant $\left(K_{e p}\right)$ and the size of the EES (Ve); whereas, semi-quantitative parameters include initial area under the gadolinium concentration-time curve (IAUGC) and time-to-peak (TTP). Non-model based parameters (IAUGC and TTP) are easy to calculate however they have a complicated and incompletely defined relationship with underlying tumor physiology. On the other hand, model-based parameters (e.g. $\mathrm{K}_{\text {trans }}, \mathrm{K}_{\mathrm{ep}}, \mathrm{V}_{\mathrm{e}}$ ) are in theory more "physiologically meaningful" and are independent of acquisition protocol and solely reflect tissue characteristics (12).

\section{Objectives}

There were three objectives of this study: 1) to acquire the quantitative and semi-quantitative pharmacokinetic parameters of the myometrium, endometrium, and cervix from the DCE-MRI of the healthy volunteers; 2) to compare the pharmacokinetic parameters of uterine fibroids with normal myometrium and;3) to determine the cut-off values for DCE-MRI parameters to differentiate between fibroids and normal myometrium.

\section{Patients and Methods}

\subsection{Patient and Healthy Volunteer Enrollment}

This study was evaluated and approved by the medical ethics committee of the University of Malaya Medical Centre, Kuala Lumpur, Malaysia. Written informed consents were obtained from all the recruited subjects. This prospective study involved two subject groups: healthy volunteers (control) and uterine fibroid patients confirmed with clinical and sonographic diagnosis. Prior to the study, the principle investigator conducted a short interview and briefing with the patients. Patient demography (i.e. age, last menstrual period, gravity and pariety) and medical history were documented. The healthy volunteer subjects were recruited from 15 women who had regular menstruations and a normal or healthy uterus on sonography. Both groups underwent the same DCE-MRI pelvic scan. Myomectomy or hysterectomy was performed for all the patients after the MRI examination and the diagnosis of uterine fibroid was confirmed by histopathological results. The flow of participants through the study is presented in Figure 1.

\subsection{MRI Scanner and Imaging Protocol}

A 1.5 Tesla MRI system (Signa HDxt 1.5T, General Electric Healthcare, Milwaukee, USA) was used in this study. Routine pelvic MRI was done for patients with fibroids using T1-weighted (T1W), T2-weighted (T2W) and short T1 inversion recovery (STIR) sequences with $4^{\circ}, 8^{\circ}$ and $12^{\circ}$ flip angles. DCE-MRI was done in both groups whereby dynamic sequences were acquired in axial planes using liver acquisition with volume acceleration (LAVA) sequence with high temporal resolution. Approximately $15 \mathrm{~mL}$ of Gd-DTPA contrast (Magnevist $0.5 \mathrm{mmol} / \mathrm{mL}$, Bayer Healthcare Pharmaceuticals Inc., Germany) was injected at a rate of $4 \mathrm{~mL} / \mathrm{s}$ through a pump injector (Tyco, Mansfield, USA) followed by a $20 \mathrm{~mL}$ saline flush. Dynamic images were acquired at approximately 37 seconds following contrast injection for 20 consecutive time points with a total acquisition time of 470 seconds.

\subsection{Post-Acquisition Image Analysis}

The images were analyzed using an independent DCEMRI software (Nordic ICE, Nordic Neuro Lab, Bergen, Norway). First, T1 relaxation times for the myometrium, endometrium and cervix were referred from the user's manual provided by General Electric Healthcare, USA. The T1 relaxation time for fibroid is currently unavailable; therefore, the T1 relaxation time for fibroid was measured using MATLAB version R2014b software (MathWorks, Massachusetts, USA). The T1 relaxation times for different tissues are shown in Table 1. 


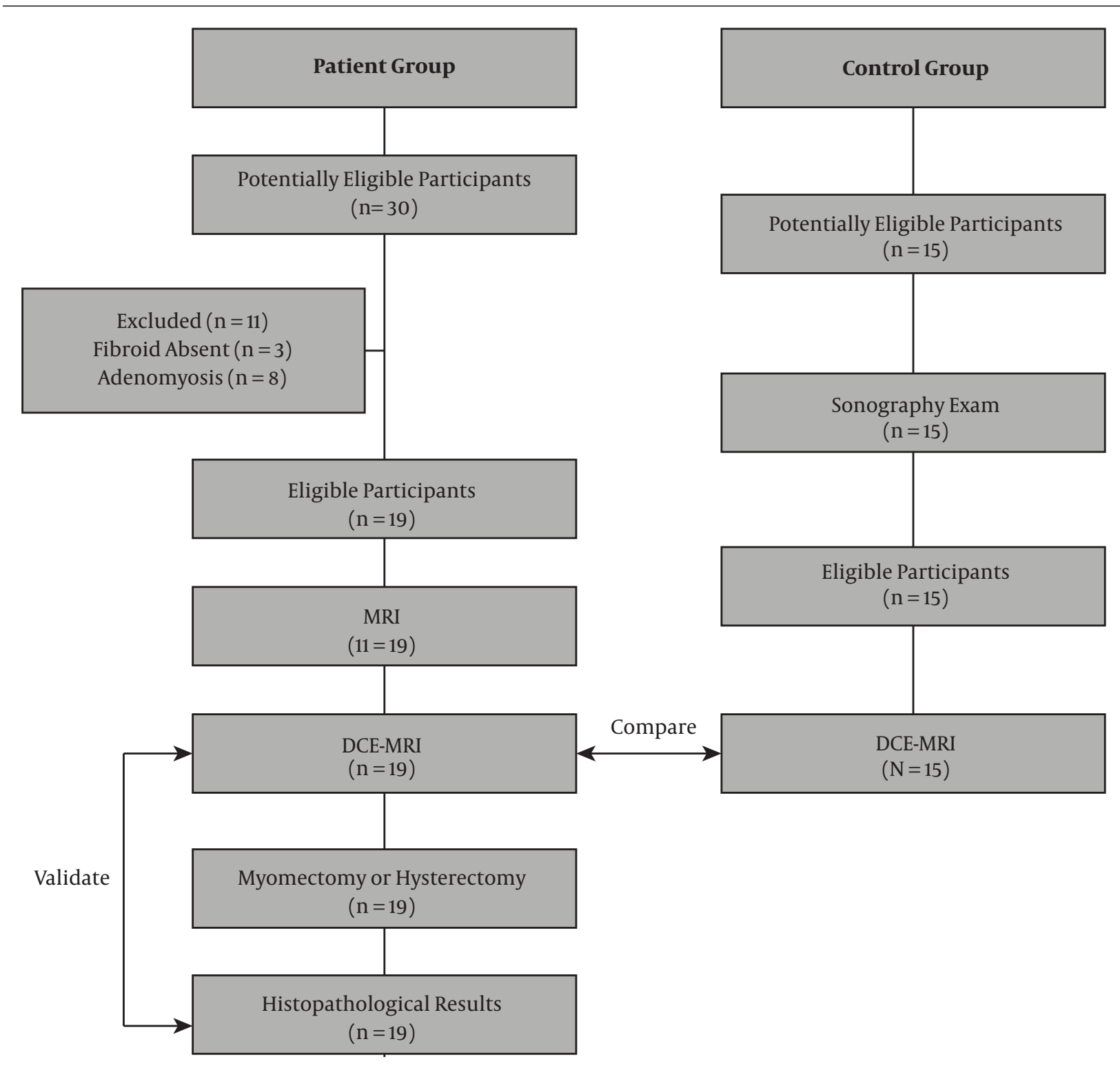

Figure 1. Flow of participants through the study 
Table 1. T1 Relaxation Times for Different Tissues

\begin{tabular}{lc}
\hline Anatomy & T1 Relaxation Time from 1.5 T MRI, (ms) \\
\hline Fibroid (calculated from MATLAB) & 740 \\
\hline Uterine myometrium $^{\mathrm{a}}$ & 1309 \\
Uterine endometrium $^{\mathrm{a}}$ & 1274 \\
Uterine cervix $^{\mathrm{a}}$ & 1135 \\
Soft tissue $^{\mathrm{a}}$ & 860 \\
\hline
\end{tabular}

${ }^{\mathrm{a}} \mathrm{T} 1$ relaxation time was obtained from the user's manual of General Electric Healthcare.

Subsequently, the pharmacokinetic parameters i.e. $\mathrm{K}_{\text {trans }}, \mathrm{K}_{\mathrm{ep}}, \mathrm{V}_{\mathrm{e}}$, IAUGC and TTP were obtained by using DCEMRI software. The descriptions of these parameters are summarized in Table 2. Images of DCE-MRI acquired at 120 flip angle were uploaded to the software and analyzed using a two compartmental pharmacokinetic model (Tofts model), which is also known as the general kinetic model. A $0.12 \mathrm{~cm}$ fixed region of interest (ROI) was placed at the myometrium, endometrium and cervix for the control group, and fibroid for the patient group. The $\mathrm{T} 1$ relaxation time for each tissue was manually put into the software (Table 1). The time-intensity curves for the respective tissues were then plotted and displayed by the software. In addition, the arterial input function (AIF) was obtained using auto-detector mode to calculate the contrast concentration in the plasma (Figure 2). The parameters were addressed both qualitatively and quantitatively using this software. The measurements for $\mathrm{K}_{\text {trans }}, \mathrm{K}_{\mathrm{ep}}, \mathrm{V}_{\mathrm{e}}$, IAUGC and TTP were repeated twice in this study.

\subsection{Statistical Analysis}

All the statistical analysis performed in this study was done using SPSS version 20.0 software (IBM Corporation, New York, USA). First, the normality of data was assessed using Shapiro-Wilk test and intraobserver variability was evaluated using intraclass correlation coefficient(ICC). The mean or median values of $\mathrm{K}_{\text {trans }}, \mathrm{K}_{\mathrm{ep}}, \mathrm{V}_{\mathrm{e}}$, IAUGC and TTP of the myometrium, endometrium and cervix were obtained and compared between the anatomical groups using Wilcoxon signed rank test. DCE-MRI parameters were then compared between the normal uterus (myometrium) and fibroids using Mann-Whitney $U$ test. A confidence interval (CI) of 95\% was used in all statistical tests. DCE-MRI parameters of the normal uterus during different menstrual cycles, i.e. follicular and luteal phases were also compared. Furthermore, the receiver operating characteristic (ROC) analysis was performed to find the representative cut-off values to differentiate uterine fibroids from normal myometrium.

\section{Results}

\subsection{Patient Characteristics}

Nineteen pre-menopausal patients (aged $40 \pm 5$ years) with the clinical diagnosis of uterine fibroids detected by ultrasound were enrolled into the study. All the cases were symptomatic. The symptoms included dysmenorrhea, hypermenorrhea and menorrhagia. No medical or interventional treatment was done on any of the patients prior to the MRI study. All the patients underwent MRI of the pelvis with the routine protocol, followed by an additional DCE-MRI scan. Either hysterectomy or myomectomy was planned for the patients based on the MRI findings. The histopathological results confirmed intramural uterine fibroids in all the patients. No complication was encountered in any of the patients during and after the examination.

\subsection{Healthy Volunteers}

The control group consisted of 15 pre-menopausal women (aged $32 \pm 6$ years) who did not have uterine fibroid confirmed by sonography. All the volunteers completed DCE-MRI of the uterus without going through the routine pelvic MRI. No complication was encountered in any of the volunteers after the study.

\subsection{Image Analysis and General Observations}

The normal uterine anatomy was best seen on T2W images where high signal intensity was observed at the endometrial canal, low signal intensity was observed at the junctional zone or inner myometrium, and intermediate signal intensity was observed at the outer myometrium. The intensity gradually enhanced on dynamic phases and the intensity at the myometrium region enhanced homogeneously. No focal enhancing lesion was observed in the control group. 
Table 2. Descriptions of the Pharmacokinetic Parameters of DCE-MRI

\begin{tabular}{ll}
\hline Parameters & Descriptions \\
\hline $\mathbf{K}_{\text {trans }}, \mathbf{m i n}^{-\mathbf{1}}$ & $\begin{array}{l}\text { Volume transfer constant for the transfer of contrast agent from the blood vessel into the extracellular extravascular space (EES). It reflects contrast } \\
\text { delivery (perfusion) and transport across the vascular endothelium (permeability). }\end{array}$ \\
$\mathbf{K}_{\mathbf{e p}}, \mathbf{m i n}^{-\mathbf{1}}$ & Flux rate constant for transfer of contrast agent back from the EES into the blood vessel \\
$\mathbf{V} \mathbf{e}$ & Proportion of EES in the tissue \\
$\mathbf{I A U G C}$ & Initial area under the gadolinium concentration-time curve \\
$\mathbf{T T P}, \mathbf{s}$ & Time-to-peak, which is the time taken for the contrast agent to reach its maximum concentration in the time-intensity curve \\
\hline
\end{tabular}

Abbreviations: DCE-MRI, dynamic contrast-enhanced magnetic resonance imaging

A

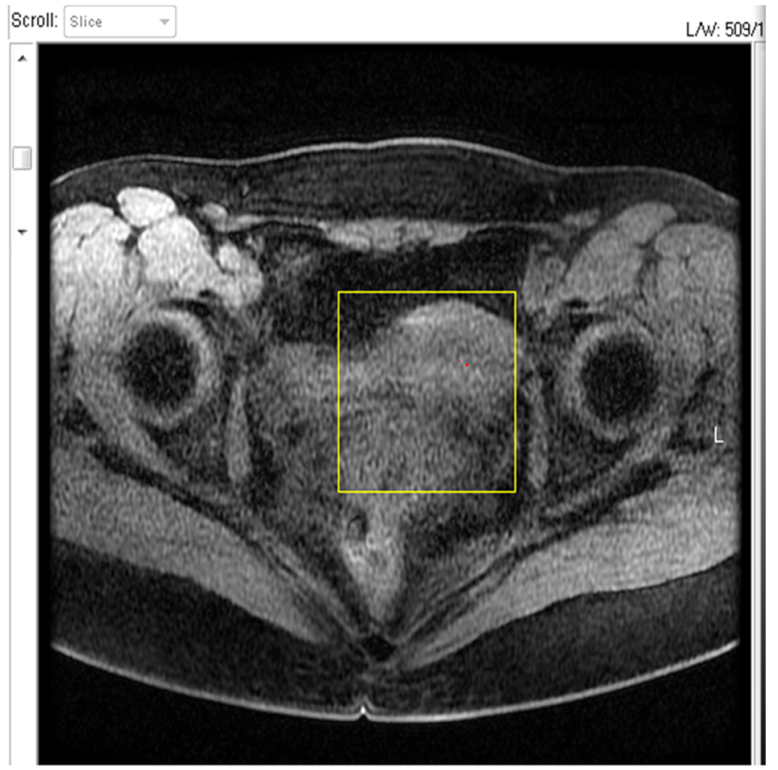

B

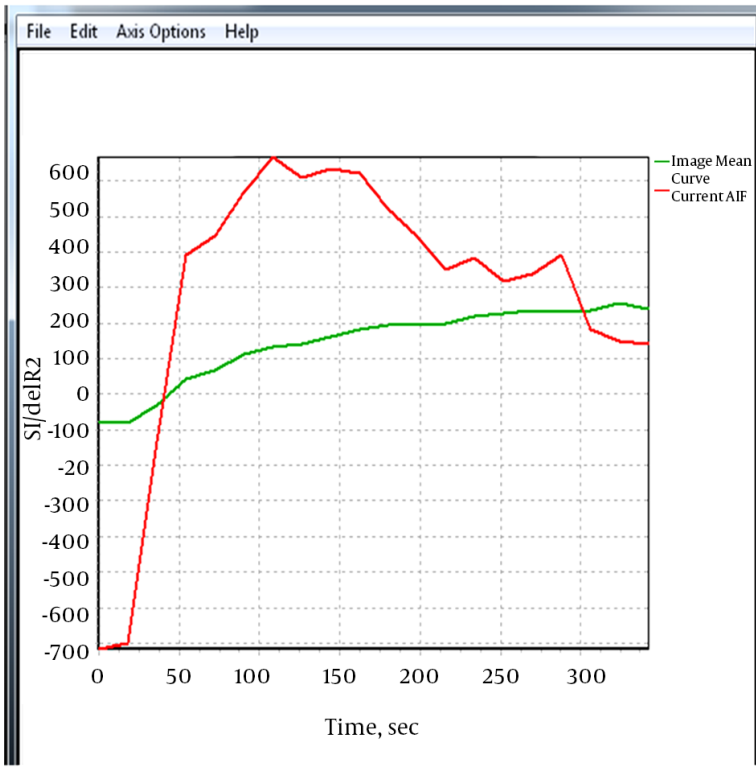

Figure 2. A, Example of an axial T1-weighted image of the pelvis with region of interest (yellow box) drawn over the uterus for quantitative dynamic contrast-enhanced magnetic resonance imaging (DCE-MRI) analysis. B, Mean signal intensity curve (green) and arterial input function (AIF) (red) for the selected tissues.

\subsection{DCE Characteristics of the Normal Uterus}

Figure 3A-D show examples of semi-quantitative maps of a normal uterus, while Figure $3 \mathrm{E}$ shows the dynamic time-intensity curve of the normal uterus. The curve has a baseline that indicates bolus arrival time followed by a gradual gradient of contrast enhancement, which was found consistent in normal tissue. The DCE parameters (median \pm standard deviation) that were then acquired from the semi-quantitative maps are presented in Table 3. The data were not normally distributed hence nonparametric analysis was used in statistical analysis. High degrees of reliability were found for intraobserver measurements (ICC $=0.757,0.980,0.990,0.750$, and 0.767 for $\mathrm{K}_{\text {trans }}, \mathrm{K}_{\mathrm{ep}}, \mathrm{V}_{\mathrm{e}}$, IAUGC, and TTP, respectively). DCE param- eters in the myometrium, endometrium and cervix were not statistically significant different $(P>0.05)$ in the control group. In addition, there was no statistically significant difference $(\mathrm{P}>0.05)$ between DCE parameters during follicular and luteal phases except for Kep in the cervix (P $=0.042)$. There was also no statistically significant difference $(P>0.05)$ between parity and all DCE parameters in the normal uterus.

\subsection{DCE Characteristics of the Fibroids}

The dynamic time-intensity curve of a uterine fibroid is shown in Figure 4. Similar to the normal uterus, the graph has a baseline followed by a gradual increment gradient corresponds to a benign lesion. The DCE-MRI parameters of 

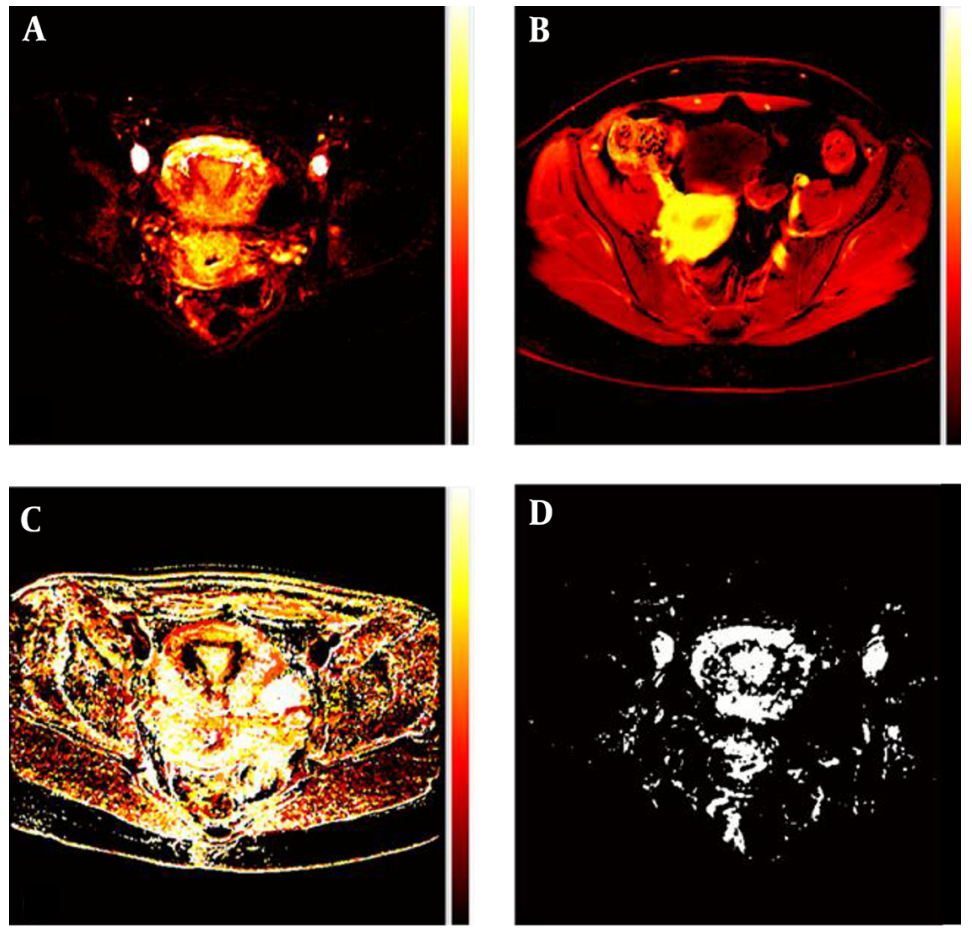

E

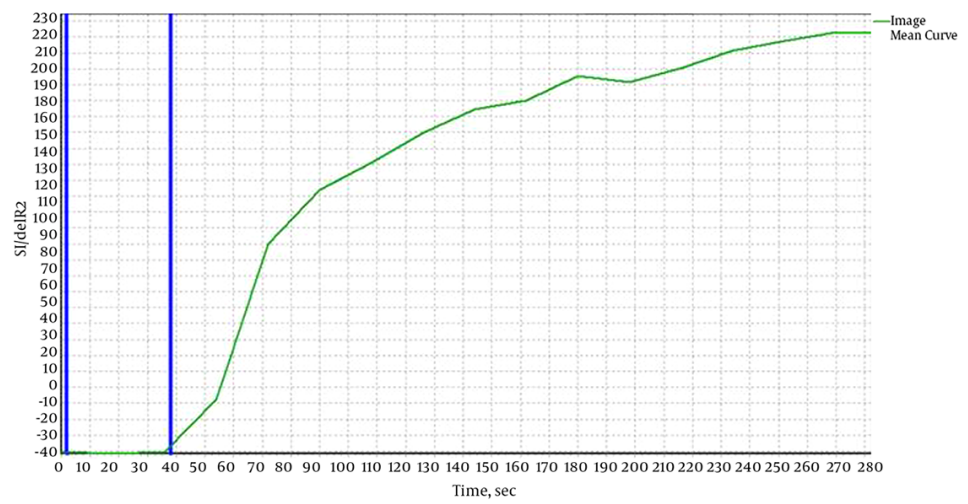

Figure 3. A-D, Semi-quantitative maps of a normal uterus acquired using the dynamic contrast enhanced magnetic resonance imaging (DCE-MRI) software. A, Ktrans; B, initial area under the gadolinium concentration time curve (IAUGC); C, time-to-peak (TTP); D, Ve. ; E, Time-intensity curve of the normal uterus. The two blue lines indicate bolus arrival time ( 37 seconds).

the uterine fibroids are presented in Table 3. Two categories of signal intensity were observed in the fibroids on T2W images where $42 \%$ of the fibroids demonstrated low signal intensity (classified as hypo-intense or "black" fibroids) and $58 \%$ of the fibroids demonstrated high signal intensity (classified as hyper-intense or "white" fibroids) (Figure 4). Some of these fibroids showed discernible signal intensity changes on $\mathrm{T} 2 \mathrm{~W}$, diffusion-weighted and T1W post-contrast images due to necrosis and degeneration. However, none of the fibroids showed delayed enhancement and no sta- tistically significant difference $(P>0.05)$ was found on the DCE-MRI parameters between black and white fibroids.

\subsection{Comparison of DCE-MRI Parameters Between the Normal} Uterus and Fibroids

In comparison of DCE-MRI parameters between normal the myometrium and fibroids, statistically significant differences were found on $\mathrm{K}_{\text {trans }}$ and $\mathrm{V}_{\mathrm{e}}(\mathrm{P}=0.014$ and $\mathrm{P}$ $=0.032$, respectively), however no statistically significant difference $(\mathrm{P}>0.05)$ was found on other parameters (Ta- 
Table 3. Median Values of DCE-MRI Parameters of the Normal Myometrium, Endometrium, Cervix and Uterine Fibroids

\begin{tabular}{|c|c|c|c|c|c|}
\hline DCE-MRI Parameters & Normal Myometrium $(n=15)$ & Endometrium $(n=15)$ & $\operatorname{Cervix}(n=15)$ & Fibroids $(n=19)$ & $\begin{array}{l}\text { P Values (Normal Myometrium } \\
\text { Versus Uterine Fibroids) }\end{array}$ \\
\hline$K_{\text {trans }}, \min ^{-1}$ & $0.26 \pm 0.13$ & $0.20 \pm 0.15$ & $0.30 \pm 0.13$ & $0.50 \pm 0.25$ & $0.014^{\mathrm{a}}$ \\
\hline$K_{e p}, \min ^{-1}$ & $0.30 \pm 0.20$ & $0.30 \pm 0.18$ & $0.30 \pm 0.07$ & $0.42 \pm 0.22$ & 0.215 \\
\hline $\mathbf{V}_{\mathbf{e}}$ & $0.50 \pm 0.24$ & $0.40 \pm 0.27$ & $0.50 \pm 0.32$ & $0.72 \pm 0.33$ & $0.032^{\mathrm{a}}$ \\
\hline IAUGC & $1.70 \pm 1.10$ & $1.81 \pm 0.82$ & $1.70 \pm 1.00$ & $15.33 \pm 0.58$ & 0.057 \\
\hline TTP, $s$ & $159.50 \pm 35.27$ & $138.50 \pm 17.71$ & $143.25 \pm 5.38$ & $137.0 \pm 47.7$ & 0.825 \\
\hline
\end{tabular}

Abbreviations: DCE-MRI, dynamic contrast-enhanced magnetic resonance imaging; IAUGC, initial area under the gadolinium concentration-time curve; TTP, time to peak; SD, standard deviation

${ }^{\mathrm{a}} \mathrm{P}<0.05$ shows a statistically significant difference.

${ }^{\mathrm{b}}$ Values are expressed as mean $\pm \mathrm{SD}$

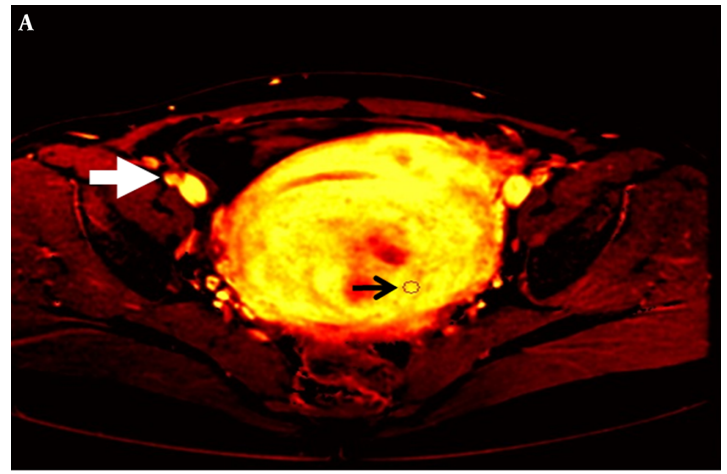

B

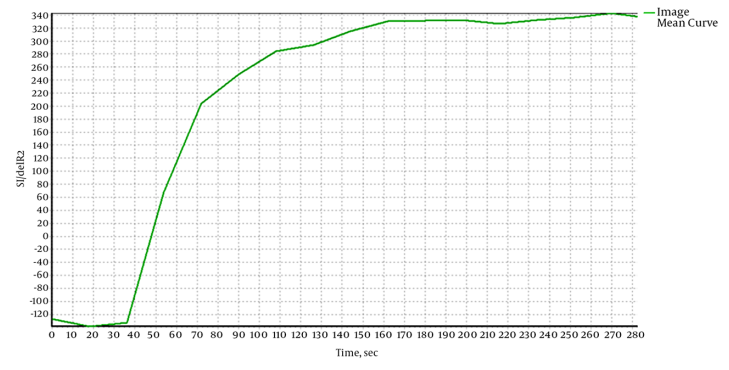

Figure 4. A and B, Time-intensity curve of an intramural uterine fibroid. The thick arrow points to the artery where arterial input function (AIF) was obtained and the thin arrow shows the region of interest (ROI) of the fibroid. Ktrans map of a patient with hyperintense (white) fibroid.

ble 3). Therefore, $\mathrm{K}_{\text {trans }}$ and $\mathrm{V}_{\mathrm{e}}$ were used in ROC analysis to determine the cut-off values for uterine fibroids. The sensitivities and specificities of different cut-off values are summarized in Table 4. In this study, both sensitivity and specificity were important, hence the cut-off value of $\mathrm{k}_{\text {trans }}$ was determined at the highest sensitivity (64.7\%) and specificity (92.3\%), which was measured at $0.47 \mathrm{~min}^{-1}$ (area under the curve $(\mathrm{AUC})=0.762,95 \% \mathrm{CI}: 0.589-0.935, \mathrm{P}=0.015)$. Using the similar method, the cut-off value of $V_{e}$ was de- termined at 0.62 which gave sensitivity and specificity of $70.6 \%$ and $78.6 \%$, respectively (AUC $=0.708,95 \%$ CI: 0.515 $0.901, \mathrm{P}=0.060)$.

\section{Discussion}

The advanced MRI techniques, such as DCE-MRI, raise the hope of providing more functional information about the diseases (13). DCE-MRI can measure angiogenesis noninvasively, and is rapidly becoming the standard method for assessing vascular properties of the lesions. For oncology assessments, this imaging technique provides additional information on the efficacy of angiogenesis inhibitors to augment evaluations based on tumor size criteria determined by the world health organization (WHO) and response evaluation criteria in solid tumors (RECIST).

The ability to analyze DCE-MRI images and accurately assess the vascular properties of the lesions provides a useful biomarker to determine a number of treatment factors. These include drug efficacy, response after chemoor radiation therapy, non-invasive distinction between benign and malignant pathology or grading of tumors, and a tool to guide lesion biopsy. DCE-MRI provides a functional approach in characterizing microvasculature, specifically perfusion and permeability of blood vessels and the volume of extracellular space (14). Abnormal microvessels in DCE-MRI may indicate hypoxia of the tissues. This is because tumor angiogenesis is associated with vessel formation and non-competent arteriovenous shunts, which lead to less effective perfusion and a more hypoxic environment than in normal tissues (15). Detecting hypoxic tumors allows hypoxia-modifying therapy or even primary surgery $(16,17)$. A study by Newbold et al. (18) demonstrated statistically significant correlation between various DCE-MRI parameters, particularly Ktrans (that shows permeability of blood vessels) and pimonidazole staining (an exogenous 
Table 4. Sensitivities and Specificities of Different Cut-Off Values for $\mathrm{K}_{\text {trans }}$ and $\mathrm{V}_{\mathrm{e}}{ }^{\mathrm{a}}$

\begin{tabular}{|c|c|c|c|c|c|}
\hline Positive If $K_{\text {trans }} \geq$ & Sensitivity (\%) & Specificity (\%) & Positive if $V_{e} \geq$ & Sensitivity (\%) & Specificity (\%) \\
\hline 0.00 & 100.0 & 0 & -0.90 & 100.0 & 0 \\
\hline 0.08 & 100.0 & 7.7 & 0.15 & 94.1 & 0 \\
\hline 0.15 & 94.1 & 23.1 & 0.25 & 82.4 & 21.4 \\
\hline 0.23 & 70.6 & 46.2 & 0.32 & 76.5 & 35.7 \\
\hline 0.28 & 70.6 & 53.8 & 0.37 & 76.5 & 42.9 \\
\hline 0.31 & 64.7 & 61.5 & 0.45 & 76.5 & 50.0 \\
\hline 0.36 & 64.7 & 76.9 & 0.52 & 70.6 & 71.4 \\
\hline 0.42 & 64.7 & 84.6 & 0.62 & 70.6 & 78.6 \\
\hline 0.47 & 64.7 & 92.3 & 0.75 & 64.7 & 78.6 \\
\hline 0.60 & 41.2 & 100.0 & 0.85 & 58.8 & 85.7 \\
\hline 0.75 & 23.5 & 100.0 & 0.95 & 29.4 & 0 \\
\hline 0.85 & 5.90 & 100.0 & 2.00 & 0 & 0 \\
\hline 1.00 & 0.0 & 100.0 & & & \\
\hline
\end{tabular}

${ }^{\mathrm{a}} 95 \%$ confidence interval is used.

marker for hypoxia). The appearance of tumor in DCEMRI has been used to successfully predict the treatment response to chemo or radiation therapy in tumors. However, quantitative DCE-MRI parameters and differences between normal uterus and abnormal myometrium with respect to the reproducibility of DCE-MRI parameters have not been previously reported to our knowledge.

This study showed that all the quantitative and semiquantitative pharmacokinetic values in uterine fibroids except TTP were higher compared to the normal myometrium. However, statistically significant differences (P $<0.05$ ) were only seen in $\mathrm{K}_{\text {trans }}$ and $\mathrm{V}_{\mathrm{e}}$. This indicated that permeability of fibroid lesions is higher than the normal tissue. The cut-off values for $\mathrm{k}_{\text {trans }}$ and $\mathrm{V}_{\mathrm{e}}$ were suggested to be $0.47 \mathrm{~min}^{-1}$ and 0.62 , respectively wherein beyond these values the uterine tissue is suspected abnormal. However, these values cannot be used to differentiate between benign and malignant masses as there was no malignant case included in this study. Further research with larger sample sizes including malignant tumors may help to differentiate benign from malignant conditions.

Among the two different types of fibroids, i.e. "black" (low signal intensity on T2W) and "white" (high signal intensity on $\mathrm{T} 2 \mathrm{~W}$ ) fibroids there was no statistically significant difference $(\mathrm{P}>0.05$ ) found on the DCE-MRI parameters between the two groups. Nevertheless, it was observed that many non-enhancing pixels were detected on the black fibroids during quantitative analysis. Previous studies have suggested that fibroids that are homogeneous and hypo-intense on $\mathrm{T} 2 \mathrm{~W}$ images seem to respond better to MR-guided focused ultrasound (MRgFUS) therapy than fibroids that are heterogeneous and hyper-intense $(19,20)$. Further, Kim et al. (21) concluded that a higher Ktrans value at baseline DCE-MRI suggested a poor ablation efficacy of MRgFUS for symptomatic uterine fibroids. Therefore, the pre-treatment DCE-MRI used in conjunction with T2W imaging may improve the criteria for selecting uterine fibroids amenable to Magnetic Resonance Guided Focused Ultrasound Surgery (MRgFUS) treatment, potentially leading to improved patient outcomes (22).

There was no statistically significant difference noted on any of the DCE-MRI parameters between different phases of menstruation (follicular and luteal phases) in the normal myometrium. Therefore, we can conclude that different phases of menstruation would not affect DCE characteristics of the tissues in DCE-MRI of the uterus. There was also no strong correlation found between DCEMRI parameters with parity factor.

Another proven contribution of DCE-MRI in pelvic imaging is its ability in distinguishing between ovarian fibroma and subserous uterine leiomyoma, which sometimes can be difficult to determine using ultrasound and computed tomography. A study carried out by Isabelle Thomassin-Naggara et al. (23) showed that DCE enhancement in uterine leiomyomas was more intense and precocious than in ovarian fibromas. Hence, the method can be used if sonography fails to show the origin of the pelvic mass.

In conclusion, uterine fibroids had significantly higher values of $K_{\text {trans }}$ and $V_{\mathrm{e}}$ compared to normal myometrium 
indicating that fibroids had higher perfusion and permeability than the normal tissues. The cut-off values of Ktrans and Ve were determined at $0.47 \mathrm{~min}^{-1}$ (sensitivity 64.7\%, specificity 92.3\%) and 0.62 (sensitivity 70.6\%, specificity 78.6\%), respectively. As previously reported, the fibroids that had a higher $\mathrm{K}_{\text {trans }}$ responded poorly to MRgFUS treatment compared to fibroids that had a lower ktrans (21). Therefore, DCE-MRI data can aid in decision making for the appropriate treatment for uterine fibroid patients. The quantitative parameters obtained from our study may provide the foundation for interpretation of DCE-MRI parameters of the uterus; however, a larger sample size is needed to validate the results.

\section{Acknowledgments}

The authors would like to thank all the patients and volunteers who participated in this study.

\section{Footnotes}

Authors' Contributions: None declared. Financial Disclosure: None declared.

Funding/Support: This study was supported in part by the University of Malaya Postgraduate Research Grant P0048/012B.

\section{References}

1. Gaustad JV, Brurberg KG, Simonsen TG, Mollatt CS, Rofstad EK. Tumor vascularity assessed by magnetic resonance imaging and intravital microscopy imaging. Neoplasia. 2008;10(4):354-62. doi: 10.1593/neo.08162.

2. Kim YE, Lim JS, Choi J, Kim D, Myoung S, Kim MJ, et al. Perfusion parameters of dynamic contrast-enhanced magnetic resonance imaging in patients with rectal cancer: correlation with microvascular density and vascular endothelial growth factor expression. Korean J Radiol. 2013;14(6):878-85. doi: 10.3348/kjr.2013.14.6.878. [PubMed: 24265562].

3. Su MY, Cheung YC, Fruehauf JP, Yu H, Nalcioglu O, Mechetner E, et al. Correlation of dynamic contrast enhancement MRI parameters with microvessel density and VEGF for assessment of angiogenesis in breast cancer. J Magn Reson Imaging. 2003;18(4):467-77. doi: 10.1002/jmri.10380. [PubMed: 14508784].

4. Jansen SA, Fan X, Karczmar GS, Abe H, Schmidt RA, Newstead GM. Differentiation between benign and malignant breast lesions detected by bilateral dynamic contrast-enhanced MRI: a sensitivity and specificity study. Magn Reson Med. 2008;59(4):747-54. doi: 10.1002/mrm.21530. [PubMed: 18383287].

5. Agner SC, Soman S, Libfeld E, McDonald M, Thomas K, Englander S, et al. Textural kinetics: a novel dynamic contrast-enhanced (DCE)-MRI feature for breast lesion classification. J Digit Imaging. 2011;24(3):44663. doi: 10.1007/s10278-010-9298-1. [PubMed: 20508965].

6. Kul S, Cansu A, Alhan E, Dinc H, Gunes G, Reis A. Contribution of diffusion-weighted imaging to dynamic contrast-enhanced MRI in the characterization of breast tumors. AJR Am J Roentgenol. 2011;196(1):210-7. doi: 10.2214/AJR.10.4258. [PubMed: 21178069].
7. Rezaei S, Rad HS. A Review Study on the Efficacy of Quantitative DCE-MRI in Breast Lesion Diagnosis. Iran J Radiol. 2014;11(1) doi: 10.5812/iranjradiol.21335.

8. Levy BS. Modern management of uterine fibroids. Acta Obstet Gynecol Scand. 2008;87(8):812-23. doi: 10.1080/00016340802146912. [PubMed: 18607823].

9. Abdullah B, Subramaniam R, Omar S, Wragg P, Ramli N, Wui A, et al. Magnetic resonance-guided focused ultrasound surgery(MRgFUS) treatment for uterine fibroids. Biomed Imaging Interv J. 2010;6(2):15. doi: 10.2349/biij.6.2.e15. [PubMed: 21611036].

10. Zahra MA, Hollingsworth KG, Sala E, Lomas DJ, Tan LT. Dynamic contrast-enhanced MRI as a predictor of tumour response to radiotherapy. Lancet Oncol. 2007;8(1):63-74. doi: 10.1016/S14702045(06)71012-9. [PubMed:17196512].

11. O'Connor JP, Jackson A, Parker GJ, Jayson GC. DCE-MRI biomarkers in the clinical evaluation of antiangiogenic and vascular disrupting agents. Br J Cancer. 2007;96(2):189-95. doi: 10.1038/sj.bjc.6603515. [PubMed: 17211479].

12. Tofts PS, Brix G, Buckley DL, Evelhoch JL, Henderson E, Knopp MV. Estimating kinetic parameters from dynamic contrast-enhanced $\mathrm{T} 1$ weighted MRI of a diffusable tracer: standardized quantities and symbols. J Magn Reson Imaging. 1999;10(3):223-32.

13. Gallagher FA. An introduction to functional and molecular imaging with MRI. Clin Radiol. 2010;65(7):557-66. doi: 10.1016/j.crad.2010.04.006. [PubMed: 20541655].

14. Bhatnagar P, Subesinghe M, Patel C, Prestwich R, Scarsbrook AF. Functional imaging for radiation treatment planning, response assessment, and adaptive therapy in head and neck cancer. Radiographics. 2013;33(7):1909-29. doi: 10.1148/rg.337125163. [PubMed: 24224586].

15. Dewhirst MW, Ong ET, Braun RD, Smith B, Klitzman B, Evans SM, et al. Quantification of longitudinal tissue pO2 gradients in window chamber tumours: impact on tumour hypoxia. BrJCancer. 1999;79(1112):1717-22. doi: 10.1038/sj.bjc.6690273. [PubMed: 10206282].

16. Overgaard J, Hansen HS, Overgaard M, Bastholt L, Berthelsen A, Specht L, et al. A randomized double-blind phase III study of nimorazole as a hypoxic radiosensitizer of primary radiotherapy in supraglottic larynx and pharynx carcinoma. Results of the Danish Head and Neck Cancer Study (DAHANCA) Protocol 5-85. Radiother Oncol. 1998;46(2):135-46. doi: 10.1016/S0167-8140(97)00220-X. [PubMed: 9510041].

17. Agrawal S, Awasthi R, Singh A, Haris M, Gupta RK, Rathore RK. An exploratory study into the role of dynamic contrast-enhanced (DCE) MRI metrics as predictors of response in head and neck cancers. Clin Radiol. 2012;67(9):1-5. doi: 10.1016/j.crad.2012.03.005. [PubMed: 22583950].

18. Newbold K, Castellano I, Charles-Edwards E, Mears D, Sohaib A, Leach $\mathrm{M}$, et al. An exploratory study into the role of dynamic contrast-enhanced magnetic resonance imaging or perfusion computed tomography for detection of intratumoral hypoxia in headand-neck cancer. Int J Radiat Oncol Biol Phys. 2009;74(1):29-37. doi: 10.1016/j.ijrobp.2008.07.039. [PubMed: 19036529].

19. Funaki K, Fukunishi H, Funaki T, Kawakami C. Mid-term outcome of magnetic resonance-guided focused ultrasound surgery for uterine myomas: from six to twelve months after volume reduction. J Minim Invasive Gynecol. 2007;14(5):616-21. doi: 10.1016/j.jmig.2007.04.009. [PubMed: 17848324].

20. Tempany CM. From the RSNA refresher courses: Image-guided thermal therapy of uterine fibroids. Radiographics. 2007;27(6):1819-26. doi: 10.1148/rg.276075096. [PubMed: 18025520].

21. Kim YS, Lim HK, Kim JH, Rhim H, Park BK, Keserci B, et al. Dynamic contrast-enhanced magnetic resonance imaging predicts immediate therapeutic response of magnetic resonance-guided high-intensity focused ultrasound ablation of symptomatic uterine fibroids. Invest Radiol. 2011;46(10):639-47. doi: 10.1097/RLI.0b013e318220785c. [PubMed: 21654495]. 
22. Yoon SW, Lee C, Kim KA, Kim SH. Contrast-Enhanced Dynamic MR Imaging of Uterine Fibroids as a Potential Predictor of Patient Eligibility for MR Guided Focused Ultrasound (MRgFUS) Treatment for Symptomatic Uterine Fibroids. Obstet Gynecol Int. 2010;2010 doi: 10.1155/2010/834275. [PubMed: 20847940].
23. Thomassin-Naggara I, Darai E, Nassar-Slaba J, Cortez A, Marsault C, Bazot M. Value of dynamic enhanced magnetic resonance imaging for distinguishing between ovarian fibroma and subserous uterine leiomyoma. J Comput Assist Tomogr. 2007;31(2):236-42. doi: 10.1097/01.rct.0000237810.88251.9e. [PubMed: 17414760]. 